\title{
Asociación entre posturas administrativas de directivos y su disposición hacia la adopción del teletrabajo
}

\author{
Omar A. Tapasco-Alzate ${ }^{(1,2) \star}$ y Jaime A. Giraldo-García(2) \\ (1) Facultad de Ciencias Exactas y Naturales, Universidad de Caldas, Calle 65 \#26-10, Manizales, Caldas, Colombia \\ (correo-e: omar.tapasco@ucaldas.edu.co) \\ (2) Facultad de Ingeniería y Arquitectura, Universidad Nacional de Colombia Sede Manizales, Carrera 27 \#64-60, \\ Manizales, Caldas, Colombia (correo-e: jaiagiraldog@unal.edu.co)
}

Recibido May. 28, 2019; Aceptado Jul. 26, 2019; Versión final Ago. 22, 2019, Publicado Feb. 2020

\begin{abstract}
Resumen
Esta investigación recopila de la literatura científica, los beneficios del teletrabajo y las dificultades evidenciadas para su adopción. Plantea como objetivo indagar en un contexto regional, entre directivos de empresas de Manizales-Colombia, cuáles barreras surgen para su implementación y sus posibles asociaciones con posturas de tipo administrativo. Para tal efecto se aplica un cuestionario entre 252 directivos, y para el tratamiento de los datos se acude al Análisis de Correspondencias Múltiples. Entre los resultados se encuentra que no son los costos de inversión ni las limitantes tecnológicas las que emergen como principales barreras; son las relacionadas con desconocimiento del tema y problemas de gestión administrativa. Se detectan perfiles directivos marcadamente diferenciados entre empresas que adoptan el teletrabajo y las que no lo hacen. Se observan también patrones de asociación entre su disposición hacia la adopción del teletrabajo y ciertas posturas administrativas. Entre estas, la desconfianza hacia esquemas organizacionales no tradicionales, la renuencia al cambio en la forma de trabajar y al uso de nuevas herramientas digitales.
\end{abstract}

Palabras clave: teletrabajo; adopción del teletrabajo; directivos; análisis de correspondencias múltiples

\section{Association between administrative positions of managers and their disposition to adopt teleworking}

\begin{abstract}
This research compiles from the scientific literature, the benefits of telework and the difficulties evidenced for its adoption. The objective is to investigate, in a regional context, among companies' managers from Manizales-Colombia, which are the barriers for its implementation and the possible associations with administrative regulations. For this purpose, a questionnaire was applied to 252 managers, and for the processing of data, Multiple Correspondence Analysis was used. Results show that neither investment costs nor technological constraints emerge as major barriers; they are those related to unfamiliarity with the subject, and management problems. Highly differentiated profiles among managers from companies adopting telework and those that do not do it. Also, patterns of association between their willingness to adopt teleworking and certain administrative positions are observed. Among these are distrust of nontraditional organizational schemes, reluctance to change the way of working and to use of new digital tools.
\end{abstract}

Keywords: telework; adoption of telework; managers; multiple correspondence analysis 


\section{INTRODUCCIÓN}

Con el advenimiento de las Tecnologías de la Información y la Comunicación (TIC) y su protagonismo a nivel mundial, han surgido diversidad de aplicaciones que abarcan los distintos contextos de la vida humana, entre ellas la posibilidad de realizar las actividades laborales sin necesidad de movilizarse a los centros de operación de las empresas, lo que se conoce como otra forma de organización laboral denominada teletrabajo. Es así como los grandes avances alcanzados en las telecomunicaciones han conducido a que dicha modalidad de trabajo haya ganado popularidad en muchas organizaciones a lo largo del mundo, aunque matizada por las diferencias culturales entre países y organizaciones, en donde la forma en que se valoran las jerarquías sociales incide en el grado de aceptación del teletrabajo (Raghuram y Fang, 2014). Adicionalmente, la necesidad evidente de generar alternativas de trabajo, han provocado que los gobiernos de muchos países hayan mostrado gran interés en poner en marcha estrategias de implementación de puestos de trabajo que hagan uso de las herramientas tecnológicas para el desempeño de sus labores. Lo que se ve evidenciado en una dinámica creciente de adopción de los programas de teletrabajo, que han introducido en los esquemas organizacionales prácticas de mayor flexibilidad para los empleados desde la perspectiva del dónde y cuándo realizar sus labores (Coenen y Kok, 2014).

Desde distintas corrientes se han difundido los múltiples beneficios asociados a esta modalidad de trabajo, tanto para el empleado como para las empresas, al igual que para la sociedad en general. Entre ellas, se señala que la práctica del trabajo a distancia incide de forma positiva en el desempeño de la organización, disminuyendo el ausentismo laboral (Davis, 2012), incrementando la estabilidad en el trabajo (Pearce, 2009) y reiterativamente se hace mención sobre la evidencia hallada en el aumento de la productividad del trabajador (Pearce, 2009; Kossek et al., 2006; Golden y Veiga, 2008; Neufeld, 2010). De igual forma, el teletrabajo les otorga a los trabajadores capacidad de autogestión, permitiéndoles trabajar a su ritmo (Pérez, 2010) y recurrir a los momentos más creativos y productivos, lo que para algunos trabajadores no corresponde a las horas regulares de trabajo (Kossek et al., 2006). Por su parte, Bailey y Kurland (2002) resaltan que entre los beneficios directamente percibidos por el teletrabajador están el dinero y el tiempo ahorrado asociados al desplazamiento a los sitios de trabajo.

Asimismo, se ha reportado una reducción significativa del estrés laboral en los teletrabajadores (Kossek et al., 2006), una disminución en el aburrimiento y la fatiga relacionada a las actividades laborales, así como una mejor conciliación entre la vida laboral y familiar (Golden, 2012). También se ha encontrado un incremento en la satisfacción de los empleados y un mayor compromiso con la organización (Coenen y Kok, 2014; Golden, 2012). Crecientemente se ha evidenciado una presión social hacia las organizaciones para que sean más "verdes", por lo que los resultados de las empresas que acogen el trabajo remoto podrían redundar en la obtención de bonos adicionales en su imagen corporativa (Harker et al., 2012). Adicionalmente ofrece una alternativa para cuando se presentan circunstancias o eventos inesperados, tales como huelgas de transporte, condiciones severas de tiempo, desastres naturales o epidemias etc. (Mello, 2007).

Entre los beneficios evidenciados para la sociedad se hace hincapié en la reducción del tráfico en las grandes ciudades, lo cual genera un impacto positivo al medio ambiente y la movilidad, con la consecuente disminución de las emisiones contaminantes al aire y de los niveles de ruido, por lo que el fomento del teletrabajo es afín a las políticas de transporte sostenible en zonas urbanas (Botzoris et al., 2016). Al igual que facilita el acceso al mercado laboral de personas con discapacidad física o que dentro de sus labores cotidianas esté el cuidado permanente de familiares (Burbach y Day, 2012). Aunque también se enuncian desventajas relacionadas con su implementación, entre las que se destacan, la sensación de aislamiento y la pérdida de visibilidad del teletrabajador hacia la organización (Burbach y Day, 2012). Además, potenciales problemas en relación a la fragmentación de las redes sociales del lugar de trabajo, conflictos al interior de los equipos de trabajo y la hostilidad entre los teletrabajadores y los no teletrabajadores (Ahmed et al., 2014). Pero a pesar de los avances tecnológicos alcanzados y los potenciales beneficios del teletrabajo, se considera que esta dinámica de adopción ha estado por debajo de las expectativas generadas (Dickson y Clear, 2006; Mello, 2007), y más aún si se contempla un contexto regional. Es por ello que este artículo indaga sobre los beneficios percibidos y las dificultades evidenciadas para su aceptación en empresas de una capital intermedia como ManizalesColombia, desde la perspectiva de los directivos, develando posibles asociaciones entre algunas de sus posturas administrativas y su disposición a acoger el trabajo remoto en sus organizaciones.

\section{OTROS ANTECEDENTES}

Además de los beneficios del teletrabajo descritos en la sección anterior, hay otros antecedentes que es necesario describir en el contexto de este trabajo: las dificultades para su adopción y la situación en Colombia

\section{Dificultades para la adopción del teletrabajo}

A pesar de la vasta literatura existente sobre los beneficios que a nivel personal, empresarial y social se han evidenciado por la puesta en marcha de dicha forma de organización laboral, las dinámicas de aceptación e 
implementación a nivel mundial han estado por debajo de las expectativas, lo que se acentúa en los países en vía de desarrollo donde el crecimiento en la acogida a esta modalidad laboral es aún muy lento y distante de las predicciones realizadas (Dickson y Clear, 2006; Mello, 2007). Es así como su implementación a gran escala aún no ha ocurrido en el mundo industrializado, lo que se convierte en un llamado a la realización de un cuidadoso análisis de todos los elementos que podrían influir en su implementación (Illegems y Verbeke 2004).

Dados los argumentos expuestos, surge la pregunta de investigación: ¿cuáles son las dificultades evidenciadas que hacen que las organizaciones empresariales no estén adoptando la modalidad del teletrabajo al ritmo esperado? Mientras que los trabajos investigativos previos se han enfocado principalmente en situaciones puntuales, al estudiar el fenómeno en determinadas organizaciones, la presente investigación analiza la generalidad del problema, detectando factores comunes y recurrentes del fenómeno y contrastándolo con resultados empíricos obtenidos por respuestas de directivos de distintos sectores empresariales en un contexto regional. Su relevancia radica en que los potenciales obstáculos para el desarrollo e implementación de un exitoso programa de teletrabajo pueden ser anticipados y planeados como parte de una aproximación estratégica global para la gestión del desempeño tanto del empleado como de la organización. Es de aclarar que se aborda la temática desde la perspectiva de los empleadores quienes son los que en primera instancia tienen el poder de decisión para implementarlo en sus organizaciones.

Al realizar una consulta detallada de la literatura, los resultados arrojan que son múltiples los factores que intervienen en la adopción del teletrabajo en las organizaciones empresariales, los cuales se enuncian a continuación. Dada la separación física ocasionada por esta forma de trabajo se generan dificultades de coordinación (Turetken et al., 2011), y al eliminarse la supervisión directa, se altera la coordinación, la comunicación y la retroalimentación (Mello, 2007). Es por ello que entre los desafíos que surgen están los relacionados con la coordinación y la organización del trabajo (Pérez et al., 2003). Consecuentemente, la evaluación de los talentos puede ser un desafío sin la supervisión directa (Burbach y Day, 2012), por lo que tanto en directivos como en empleados surge la preocupación sobre la mensurabilidad de los resultados del trabajo telerealizado (Turetken et al., 2011).

Al respecto, Mello (2007) enuncia que una de las más acuciantes preocupaciones de muchos gerentes que supervisan a sus teletrabajadores es determinar la forma de medir sus desempeño. De otra parte, el aislamiento de la cultura de la compañía experimentado por los teletrabajadores, al no estar físicamente presente, afecta sensiblemente la interacción con los colegas y supervisores (Mello, 2007). Cuando los teletrabajadores toman la ventaja de la flexibilidad en los horarios de su trabajo, ellos pueden no estar disponibles para sus compañeros quienes permanecen en horario de oficina (Pérez et al., 2003). En el mismo sentido, muchos empleados encuentran problemático el no disponer de compañeros o supervisores a quien solicitarle ayuda con decisiones difíciles o problemas que requieren de un conocimiento especializado (Topi, 2004).

De la investigación realizada por Pérez et al. (2005) se desprende también que la seguridad de la información, aunque en menor medida, sigue considerándose una barrera importante. En este sentido, Peters et al. (2004) hacen referencia a los hallazgos investigativos en donde surge como barrera principal la relacionada con los problemas de seguridad de los datos. Al respecto, Kim (2018) declara que modalidades de trabajo flexible como el trabajo realizado a la distancia no sólo incrementan las posibilidades de pérdida o robo de información sino que aumenta la vulnerabilidad de los equipos de cómputo al ataque de software malicioso. Con respecto al acceso a las tecnologías, las organizaciones enfrentan principalmente tres desafíos tecnológicos: conectividad, transferencia de información y comunicación (Pearce, 2009), por lo que los empresarios serán más renuentes a implementar el trabajo remoto en sus organizaciones cuando hay carencias de los recursos requeridos (Pérez et al., 2005), dado que la efectividad del teletrabajo demanda equipos y software en mayor cantidad, más rápidos y mejores (Davis, 2012).

De otra parte, la libertad que conlleva el trabajo a distancia, implica una responsabilidad, la de saber administrarla correctamente (Pérez, 2010). Ahmed et al. (2014) hacen referencia al impacto que a nivel de satisfacción, desempeño y productividad de los teletrabajadores tienen su experiencia en el trabajo, sus habilidades comunicativas y su independencia en la realización de las tareas. Los empleados más talentosos en el uso de las tecnologías de la información y la comunicación son aquellos quienes con más frecuencia teletrabajan y/o tienen más altas probabilidades de vinculación a estrategias de trabajo a distancia ofrecidas en las compañías (Peters et al., 2004). Del lado de los directivos, una de las razones manifiestas que explican el estancamiento de esta modalidad de trabajo es el ambiente de desconfianza por parte de los empleadores, quienes con frecuencia expresan el temor de permitirles a sus empleados que trabajen desde sus casas, lejos de la supervisión de sus jefes directos (Bloom et al., 2014). 
A este último respecto Mello (2007) expresa que la razón más común para el fracaso de los programas de teletrabajo es la resistencia de los administrativos, y Kirs y Bagchi (2012) enuncian que dicha preocupación es generada por la falta de confianza hacia los teletrabajadores cuando están por fuera del alcance físico. Por su parte, Illegems y Verbeke (2004) encontraron diferencias significativas entre empresas adoptadoras y no adoptadoras en las percepciones de sus empleados, sugiriendo que estos últimos carecen de confianza en las prácticas administrativas de recursos humanos de la empresa para adaptarse apropiadamente a los cambios requeridos por el medio. Un elemento explicativo de ello es la importancia que los directivos le dan a tener un control total sobre los procesos de trabajo en sus áreas. Sin embargo con frecuencia los directivos rechazan cambiar sus hábitos de coordinación y control, lo cual sugiere una barrera organizacional sustancial existente para la implementación de dicha forma de organización laboral (Bailey y Kurland, 2002; Illegems y Verbeke, 2004).

Dado que la toma de decisiones en las organizaciones puede estar afectada por el miedo que se desprende del desconocimiento y la incertidumbre, la percepción gerencial se convierte en un aspecto determinante, por lo que se hace necesario tener evidencia de que estas nuevas formas de trabajar como el trabajo remoto son realmente más efectivas para las organizaciones que las estrategias tradicionales (Harker et al., 2012). A este respecto, Peters y Batenburg (2015) acotan que las tácticas de gestión orientadas a resultados pueden ser alineadas de forma más natural a la acogida de una política formal de teletrabajo, además de ser una de las posibles acciones emprendidas por la organización para mitigar las consecuencias negativas del trabajo remoto.

Por otra parte, Peters y Dulk (2003) manifiestan que la mayoría de académicos en el campo se enfocan en los cambios organizacionales y las prácticas administrativas que parecen ser necesarias para el acogimiento de esta forma de organización laboral y les dan menos atención a las cuestiones de si estas condiciones son igualmente compatibles en diferentes culturas nacionales; encontrando diferencias en las tasas de admisión del teletrabajo en países europeos atribuibles a diferencias culturales y exponen que las culturas del norte europeo se caracterizan por tener una propensión a evitar fuertemente la incertidumbre y tener distanciamientos cortos de poder entre directivos y empleados, lo que favorece la implantación de arreglos de trabajo flexible, mientras que los países del sur europeo se caracterizan por lo opuesto, lo que explica las diferencias registradas en las tasas de adopción a favor de los países del norte. A continuación se presenta una tabla resumen (Tabla 1) de las razones expuestas por los investigadores referenciados sobre las evidencias encontradas de la problemática emergente para la aceptación de dicha modalidad laboral en las empresas.

\section{El teletrabajo en Colombia}

Según el más reciente informe de penetración del teletrabajo en Colombia, el número de teletrabajadores ha alcanzado la cifra de 122.278 en 2018 , es decir, que se ha cuadruplicado la cifra de 2012 , en el que se reportaron 31.553 teletrabajadores, concentrados principalmente en las grandes capitales, ya que los tres mayores centros urbanos, Bogotá, Medellín y Cali, captan el $87.6 \%$ de toda la población de teletrabajadores del país. Se sabe además que $71.5 \%$ están vinculados a PYMES, $21.2 \%$ a medianas empresas y $7.3 \%$ a grandes empresas. Con respecto a la modalidad escogida, $37 \%$ han optado por el teletrabajo total o autónomo, $34 \%$ bajo el modelo mixto o suplementario y el restante $29 \%$ por la modalidad móvil. Con relación al sitio donde realizan sus actividades laborales, una amplia mayoría, $74 \%$ de teletrabajadores, lo hacen desde su hogar o domicilio (Corporación Colombia Digital-MinTic, 2018). Por su parte, el estudio del CCITFedesarrollo (2014) realizado entre empresarios en las ciudades de Bogotá, Medellín, Cali y Barranquilla, enuncia como el principal obstáculo para incrementar la penetración del trabajo a distancia en el país, la alta aversión al cambio heredada de los esquemas organizacionales tradicionales y en segunda instancia a las limitaciones tecnológicas en las empresas. Puntualmente se encontró que dos de cada tres directivos de empresas medianas creen que el trabajo a distancia repercute en una desvinculación laboral que puede traducirse fácilmente en dificultades de monitoreo del personal de la empresa.

En esta investigación se presentan los resultados empíricos de las respuestas dadas por directivos de distintos sectores empresariales de la ciudad de Manizales (Colombia) a algunos cuestionamientos directos sobre las dificultades evidenciadas para la aceptación del trabajo a distancia en un contexto regional, de las cuales se desprende que la principal causa es el desconocimiento, seguido de la resistencia al cambio, los problemas del control del teletrabajador, el temor a la incertidumbre y la seguridad de la información. De igual forma se recoge y categorizan las dificultades que han surgido para su puesta en marcha alrededor del mundo, entre las que se destacan algunas razones que atañen a la necesidad de adoptar formas alternativas de gestión administrativa y otras relacionadas con la necesidad de reforzar los lazos de confianza entre empleados y administrativos, dificultades que están matizadas por aspectos culturales del entorno. Finalmente se exponen los resultados del Análisis de Correspondencias Múltiples (ACM), el cual arroja que entre los directivos de las empresas que adoptan el trabajo remoto y las que no, existen unas claras diferencias con relación a sus posturas hacia distintos aspectos de gestión administrativa. 
Tabla 1. Resumen de los argumentos referenciados en las dificultades para la adopción del teletrabajo

\begin{tabular}{|c|c|c|}
\hline Dificultades & Argumentos & Referencias \\
\hline $\begin{array}{l}\text { Pérdida de control sobre } \\
\text { los teletrabajadores }\end{array}$ & $\begin{array}{l}\text { La separación física ocasionada por la } \\
\text { realización del trabajo remoto genera } \\
\text { dificultades en la coordinación y pérdida de } \\
\text { control sobre los empleados. }\end{array}$ & $\begin{array}{l}\text { Bailey y Kurland (2002), Peters y Dulk } \\
\text { (2003), Pérez et al. (2005), Mello } \\
\text { (2007), Pérez y Gálvez (2009), } \\
\text { Turetken et al. (2011), Dutcher (2012) }\end{array}$ \\
\hline $\begin{array}{l}\text { Medición del desempeño } \\
\text { de los teletrabajadores }\end{array}$ & $\begin{array}{l}\text { Dado que los métodos de valoración del } \\
\text { desempeño tradicionales se basan en la } \\
\text { supervisión presencial, se hace necesaria } \\
\text { formas alternativas de evaluación que apunten } \\
\text { al cumplimiento de objetivos. }\end{array}$ & $\begin{array}{l}\text { Topi (2004), Dickson y Clear (2006), } \\
\text { Mello (2007), Pearce (2009), Burbach } \\
\text { y Day (2012) }\end{array}$ \\
\hline $\begin{array}{l}\text { Impacto sobre el equipo de } \\
\text { trabajo }\end{array}$ & $\begin{array}{l}\text { Al no estar el empleado físicamente presente, } \\
\text { se afecta sensiblemente la interacción con los } \\
\text { colegas y supervisores. }\end{array}$ & $\begin{array}{l}\text { Topi (2004), Pérez et al. (2005), Mello } \\
\text { (2007), Pearce (2009), Davis (2012) }\end{array}$ \\
\hline $\begin{array}{l}\text { Seguridad de la } \\
\text { información }\end{array}$ & $\begin{array}{l}\text { Los riesgos de extracción ilegal de información } \\
\text { confidencial se incrementan cuando los } \\
\text { empleados tienen acceso desde sus } \\
\text { residencias a dicha información. }\end{array}$ & $\begin{array}{l}\text { Peters et al. (2004), Pérez et al. } \\
\text { (2005), Dickson y Clear (2006), Mello } \\
\text { (2007), Kim (2018) }\end{array}$ \\
\hline $\begin{array}{l}\text { Costos de implementación } \\
\text { y acceso a las tecnologías }\end{array}$ & $\begin{array}{l}\text { Para el buen desarrollo de un programa de } \\
\text { implementación es indispensable disponer de } \\
\text { las herramientas tecnológicas apropiadas. }\end{array}$ & $\begin{array}{l}\text { Pérez et al., 2005), Pearce (2009), } \\
\text { Valmohammadi (2012) }\end{array}$ \\
\hline $\begin{array}{l}\text { Selección de empleados } \\
\text { aptos }\end{array}$ & $\begin{array}{l}\text { Un buen trabajador no es necesariamente un } \\
\text { buen teletrabajador, es primordial que posea } \\
\text { ciertas habilidades y conocimientos, así como } \\
\text { buena disposición hacia el teletrabajo. }\end{array}$ & $\begin{array}{l}\text { Peters et al. (2004), Pérez et al. } \\
\text { (2005), Mello (2007), Turetken et al. } \\
\text { (2011), Burbach y Day (2012), Ahmed } \\
\text { et al. (2014) }\end{array}$ \\
\hline $\begin{array}{l}\text { Rechazo entre los } \\
\text { empleadores }\end{array}$ & $\begin{array}{l}\text { Desconfianza hacia el comportamiento de los } \\
\text { empleados por fuera de la supervisión física. } \\
\text { Preocupación de que los desafíos de } \\
\text { coordinación incrementen su carga laboral. }\end{array}$ & $\begin{array}{l}\text { Pérez et al. (2005), Turetken et al. } \\
\text { (2011), Mello (2007), Neufeld et al. } \\
\text { (2010), Bloom et al., (2012), Kirs y } \\
\text { Bagchi (2012) }\end{array}$ \\
\hline $\begin{array}{l}\text { Resistencia al cambio de } \\
\text { las estructuras } \\
\text { organizacionales }\end{array}$ & $\begin{array}{l}\text { Implica un cambio de perspectiva en la cultura } \\
\text { del trabajo tradicional soportada en la } \\
\text { presencialidad. }\end{array}$ & $\begin{array}{l}\text { Illegems y Verbeke (2004), Pérez et } \\
\text { al. (2005) }\end{array}$ \\
\hline Temor a la incertidumbre & $\begin{array}{l}\text { El desconocimiento sobre el concepto de } \\
\text { teletrabajo y/o sus beneficios potenciales } \\
\text { conlleva a tener recelo en su adopción. }\end{array}$ & $\begin{array}{l}\text { Peters y Dulk (2003), Pérez et al. } \\
\text { (2005), Dickson y Clear (2006) }\end{array}$ \\
\hline Aspectos culturales & $\begin{array}{l}\text { Las dinámicas de adopción también se ven } \\
\text { matizadas por aspectos culturales. }\end{array}$ & $\begin{array}{l}\text { Peters y Dulk (2003), Topi (2004), } \\
\text { Valmohammadi (2012) }\end{array}$ \\
\hline
\end{tabular}

\section{METODOLOGÍA}

Aunque no hay consenso en la literatura sobre la definición del teletrabajo y sus distintas connotaciones, tales como trabajo remoto, trabajo a distancia o teleconmutación, entre otras, la presente investigación se acoge a la definición genérica dada por Pérez et al. (2003), en la que expresa que es una actividad en la que surgen algunas características comunes tales como el hacer uso parcial o completo de las tecnologías de la información y las telecomunicaciones permitiéndole a los trabajadores tener acceso a sus actividades laborales desde distintas y remotas locaciones Con el propósito de indagar sobre las dificultades que a nivel regional surgen entre los directivos para el acogimiento de formas flexibles de laborar, como lo es el trabajo remoto, en sus organizaciones, se construyó un instrumento que recoge la información arrojada de la revisión bibliográfica sobre la temática en distintos contextos geográficos a nivel mundial.

Para el levantamiento de la información se acude a la adquisición del registro de empresas a través de la Cámara de Comercio de Manizales, teniendo como población objetivo las organizaciones que se desempeñan en los sectores económicos más consistentes con la naturaleza de las actividades a ser telerealizadas (Pérez et al., 2003). Por lo cual, y acudiendo a la clasificación asignada en el CIIU Rev. 3.1 A.C.- Clasificación Industrial Internacional Uniforme de todas las actividades económicas (DANE, 2006), se seleccionan los sectores clasificados en las categorías $\mathrm{C}, \mathrm{D}, \mathrm{G}, \mathrm{H}, \mathrm{J}, \mathrm{K}, \mathrm{M}, \mathrm{O}, \mathrm{P}, \mathrm{Q}$ y $\mathrm{S}$, es decir, sector industrial manufacturero, energético, comercio, transporte, información y comunicación, actividades financieras, actividades profesionales y técnicas, administración pública, educación, salud y otras actividades de servicios, respectivamente. Para tal efecto, se realizó un muestreo aleatorio simple, que a un nivel de confianza del $95 \%$, un margen de error del $4 \%$ y estimando que al menos un $90 \%$ de los directivos sabían que era el teletrabajo, arrojó un tamaño muestral de 216 empresas. Y presumiendo una tasa de respuesta del $80 \%$, se ajustó el muestreo a 270 empresas. Se realizaron visitas personales a los directivos para la aplicación de los 
instrumentos, obteniéndose un total de 252 respuestas, lo que equivale a una tasa de respuestas efectiva de 93.3\%. En la medición de fiabilidad se recurre al alfa de Cronbach, obteniéndose para estos datos un valor de 0.716, el cual es considerado un indicador aceptable de fiabilidad (Harker et al., 2012).

Posteriormente y recurriendo al uso del software licenciado SPSS versión 22 y al Excel, se realiza un análisis descriptivo de los datos obtenidos, conducentes a la descripción del conocimiento sobre el trabajo remoto y los beneficios y las dificultades que los directivos perciben para su implantación. Seguidamente se acude a una Análisis de Correspondencias Múltiples (ACM), cuyo objetivo se centra en descubrir estructuras de relaciones de variables predominantemente categóricas en búsqueda de posibles explicaciones para las distintas posturas expresadas de los empresarios hacia dicha modalidad laboral. Para lo cual se realiza una categorización de las respuestas obtenidas en una variable que hemos denominado "Disposición hacia el Teletrabajo", de la siguiente manera: el grupo de directivos que sabían que era el teletrabajo se dividió en dos, los que ya lo habían implementado o tenían planeado realizarlo a corto plazo y los que no pensaban hacerlo a corto plazo; y el grupo que manifestaba desconocimiento acerca de dicho concepto, también se dividió en dos, los que consideraban que posiblemente si lo implementarían en el futuro después de enterarse de que trataba el mismo, y los que no lo veían como una opción factible para sus empresas. De igual forma, se procede con un grupo de preguntas realizadas a los encuestados, tal y como queda descrito en la Tabla 2, como se muestra a continuación.

Tabla 2: Categorización de las variables para el ACM

\begin{tabular}{|c|c|c|c|}
\hline Pregunta & Variable & Categorías & Notación \\
\hline \multirow{3}{*}{ Número de trabajadores que laboran en la empresa } & \multirow{3}{*}{$\begin{array}{l}\text { Número } \\
\text { Trabajadores }\end{array}$} & Menos de 11 & $<11$ \\
\hline & & Entre 11 y 50 & $11 \mathrm{a} 50$ \\
\hline & & Más de 50 & $>50$ \\
\hline \multirow{2}{*}{ ¿Hace uso de herramientas de almacenamiento en la nube? } & \multirow{2}{*}{ Nube } & No & Nnube \\
\hline & & Si & Snube \\
\hline \multirow{2}{*}{$\begin{array}{l}\text { ¿En el último año, la empresa ha realizado cursos de } \\
\text { capacitación en competencias informáticas a sus } \\
\text { trabajadores? }\end{array}$} & \multirow{2}{*}{ Capacitación } & No & Ncap \\
\hline & & $\mathrm{Si}$ & Scap \\
\hline \multirow{2}{*}{$\begin{array}{l}\text { ¿Cuenta la entidad con algún software que permita } \\
\text { monitorear y hacer seguimiento a las actividades de los } \\
\text { empleados? }\end{array}$} & \multirow{2}{*}{ Monitoreo } & No & Nmon \\
\hline & & $\mathrm{Si}$ & Smon \\
\hline \multirow{2}{*}{$\begin{array}{l}\text { ¿En su organización se promueve la participación de los } \\
\text { trabajadores a través de un proceso de toma de decisiones } \\
\text { descentralizadas? }\end{array}$} & \multirow{2}{*}{ Decisiones } & No & Ndecis \\
\hline & & $\mathrm{Si}$ & Sdecis \\
\hline \multirow{2}{*}{ En su organización existe la figura de supervisor } & \multirow{2}{*}{ Supervisor } & No & Nsup \\
\hline & & $\mathrm{Si}$ & Ssup \\
\hline \multirow{2}{*}{$\begin{array}{l}\text { La evaluación de los talentos puede ser un desafío sin la } \\
\text { supervisión directa }\end{array}$} & \multirow{2}{*}{$\begin{array}{l}\text { Supervisión } \\
\text { Directa }\end{array}$} & No & NsupDir \\
\hline & & $\mathrm{Si}$ & SsupDir \\
\hline \multirow{2}{*}{$\begin{array}{l}\text { Los riesgos de extracción ilegal de información confidencial } \\
\text { se incrementan cuando los empleados tienen acceso desde } \\
\text { sus casas a dicha información }\end{array}$} & \multirow{2}{*}{$\begin{array}{l}\text { Riesgos } \\
\text { Seguridad }\end{array}$} & No & NRiSeg \\
\hline & & $\mathrm{Si}$ & SRiSeg \\
\hline \multirow{2}{*}{ Estoy habituado a mi forma de trabajar y no deseo cambiarla } & \multirow{2}{*}{$\begin{array}{l}\text { Cambiar } \\
\text { Forma } \\
\text { Trabajar }\end{array}$} & No & NCamFT \\
\hline & & $\mathrm{Si}$ & SCamFT \\
\hline \multirow{2}{*}{ El proceso del trabajo debería ser visible para su supervisión } & \multirow{2}{*}{$\begin{array}{l}\text { Trabajo } \\
\text { Visible }\end{array}$} & No & NTrabVis \\
\hline & & $\mathrm{Si}$ & STrabVis \\
\hline \multirow{2}{*}{$\begin{array}{l}\text { En general, cuando usted hace negocios con otras personas } \\
\text { que no conoce considera que se puede ser: }\end{array}$} & \multirow{2}{*}{ Negocios } & Muy/Confiado & Confiado \\
\hline & & Muy/Cuidadoso & Cuidadoso \\
\hline \multirow{4}{*}{ Disposición hacia el teletrabajo } & \multirow{4}{*}{$\begin{array}{l}\text { Disposición } \\
\text { Teletrabajo }\end{array}$} & Lo ha implementado & SiTT \\
\hline & & $\begin{array}{l}\text { No al teletrabajo a } \\
\text { corto plazo }\end{array}$ & NoTTcp \\
\hline & & $\begin{array}{l}\text { Quizás lo adopte en } \\
\text { el futuro }\end{array}$ & QuizasTT \\
\hline & & $\begin{array}{l}\text { No considero } \\
\text { adoptarlo }\end{array}$ & NoTT \\
\hline
\end{tabular}




\section{RESULTADOS Y DISCUSIÓN}

Los porcentajes por sectores empresariales de los 252 directivos encuestados fueron: Actividades Profesionales y Técnicas $24.2 \%$, Información y Comunicaciones $21 \%$, Industria $11.5 \%$, Comercial $11.1 \%$, Educativo 7.9\%, Administración Pública 6\%, Actividades Financieras 5.2\%, Salud 3.2\%, Energético 2\%, Transporte $1.6 \%$ y Otras Actividades de Servicios $6.3 \%$. Según el número de empleados, $48 \%$ son de empresas con menos de 11 trabajadores, $19.8 \%$ entre 11 y 50 y $32.1 \%$ con más de 50 . Entre las respuestas dadas se tiene que $77.7 \%$ de los directivos hacen uso de herramientas de almacenamiento en la nube, $41.8 \%$ de las empresas ha realizado cursos de capacitación en competencias informáticas a sus trabajadores, 56.7 $\%$ manifiesta disponer de algún software para el monitoreo de las actividades laborales de sus empleados, $13.9 \%$ promueve con frecuencia la participación de los empleados en la toma de decisiones descentralizadas, y $73 \%$ lo promueve en ocasiones, en $79.8 \%$ de las empresas existe la figura de supervisor, $39.3 \%$ de los directivos expresa ser una persona confiada cuando realiza negocios con otras personas que no conoce, mientras que el restante $60.7 \%$ manifiesta ser cuidadoso al respecto. Con relación al conocimiento que se tiene del concepto de teletrabajo, $34.5 \%$ de los directivos dicen no saber qué es, ver Figura 1. Una prueba de independencia arroja que este desconocimiento no está asociado al tamaño de la empresa ( $\mathrm{P}=0.378)$, es decir, que el desconocimiento es similar en directivos de empresas grandes, medianas o pequeñas.

Si

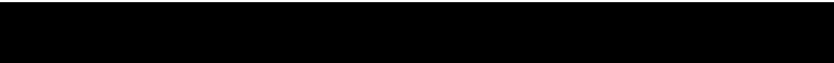

47,2

He escuchado el término pero no lo tengo claro

No

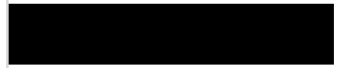

18,3
34,5

Fig. 1: Resultados a la pregunta: ¿Sabe usted qué es el teletrabajo?

Entre los que respondieron si saber qué era el teletrabajo se encontró que en 13 empresas ya lo han implementado y en 16 de ellas consideran implementarlo a corto plazo. Mientras que entre los 87 que respondieron no saber sobre dicho concepto, y después de explicárseles en qué consistía y cuál era la normativa que lo regía, 54 expresaron que contemplarían considerar al teletrabajo como una alternativa de trabajo para sus empleados. Con relación a la pregunta central de la investigación, en la Figura 2 se plasman los resultados de las respuestas obtenidas, en donde sobresale el desconocimiento sobre el tema como la principal dificultad a la que se enfrentan los directivos indagados para la implantación de dicha práctica laboral en sus empresas. Y cabría preguntarse si la misma razón es compartida entre directivos empresariales de otras capitales regionales, en donde la penetración del trabajo a distancia muestra indicadores muy bajos, en comparación con las grandes capitales, lo que requeriría más evidencia empírica.

Además de ser un aspecto no mencionado en la revisión de literatura, lo anterior se contrapone a la gran dinámica de promoción de dicha forma de organización laboral adelantada por el gobierno nacional, liderada por los Ministerios de Trabajo y de las TIC. Es así como se crea el decreto 884 de 2012, reglamentado por la ley 1221 de 2008, la cual brinda las pautas normativas para que el teletrabajador tenga iguales garantías que el trabajador que realiza sus labores en forma presencial, proyectos tales como: "El Pacto por el Teletrabajo", firmado por distintas entidades del sector público y privado en diferentes regiones del país para promover la cultura del trabajo remoto, la guía sobre la implantación del mismo que ofrecen el Ministerio de Trabajo, el Ministerio TIC y la Corporación Colombia Digital a través del "Libro Blanco: el ABC del teletrabajo en Colombia" y los distintos acompañamientos propuestos por los Ministerios canalizados a través de las Cámaras de Comercio de las ciudades capitales.

Lo anteriormente mencionado podría llevar a pensar sobre la necesidad de abarcar otros canales de comunicación conducentes a una divulgación más efectiva, o que quizás dicho trabajo de difusión se ha concentrado en las grandes capitales, pero ha sido insuficiente en las regionales, lo que también iría en concordancia con las diferencias en los porcentajes de penetración generados entre estos dos tipos de ciudades. Igualmente, se hace necesario difundir experiencias exitosas cercanas que les proporciones herramientas de juicio a los directivos para la toma de decisiones conducentes a la adopción de esta modalidad laboral.

De otro lado, y en concordancia con los hallazgos obtenidos en la revisión bibliográfica, otros aspectos tales como la resistencia al cambio entre los directivos, la pérdida de control sobre el empleado, el temor a la incertidumbre, los posibles riesgos de seguridad de la información y los posibles efectos negativos para los equipos de trabajo, surgen como las barreras más relevantes para implementar el teletrabajo en sus 
organizaciones. Se destaca además que sólo $9.6 \%$ de los directivos manifiesta su preocupación por la inversión necesaria para la puesta en funcionamiento de dicha modalidad laboral. Por lo que los resultados son consistentes con los hallazgos de Dickson y Clear (2006), quienes expresan que las principales influencias inhibidoras para la adopción y difusión del teletrabajo están más relacionadas con el ser humano y la organización que con los factores tecnológicos.

A la pregunta sobre los incentivos que podría el gobierno dar para promover el teletrabajo, la gran mayoría propone el tener beneficios fiscales a las empresas que lo implementen y cerca de una cuarta parte reseña la necesidad de tener asesoría en el proceso de implementación, lo que refuerza la idea antes mencionada sobre la necesidad de implementar estrategias de divulgación más efectivas por parte de los entes gubernamentales.

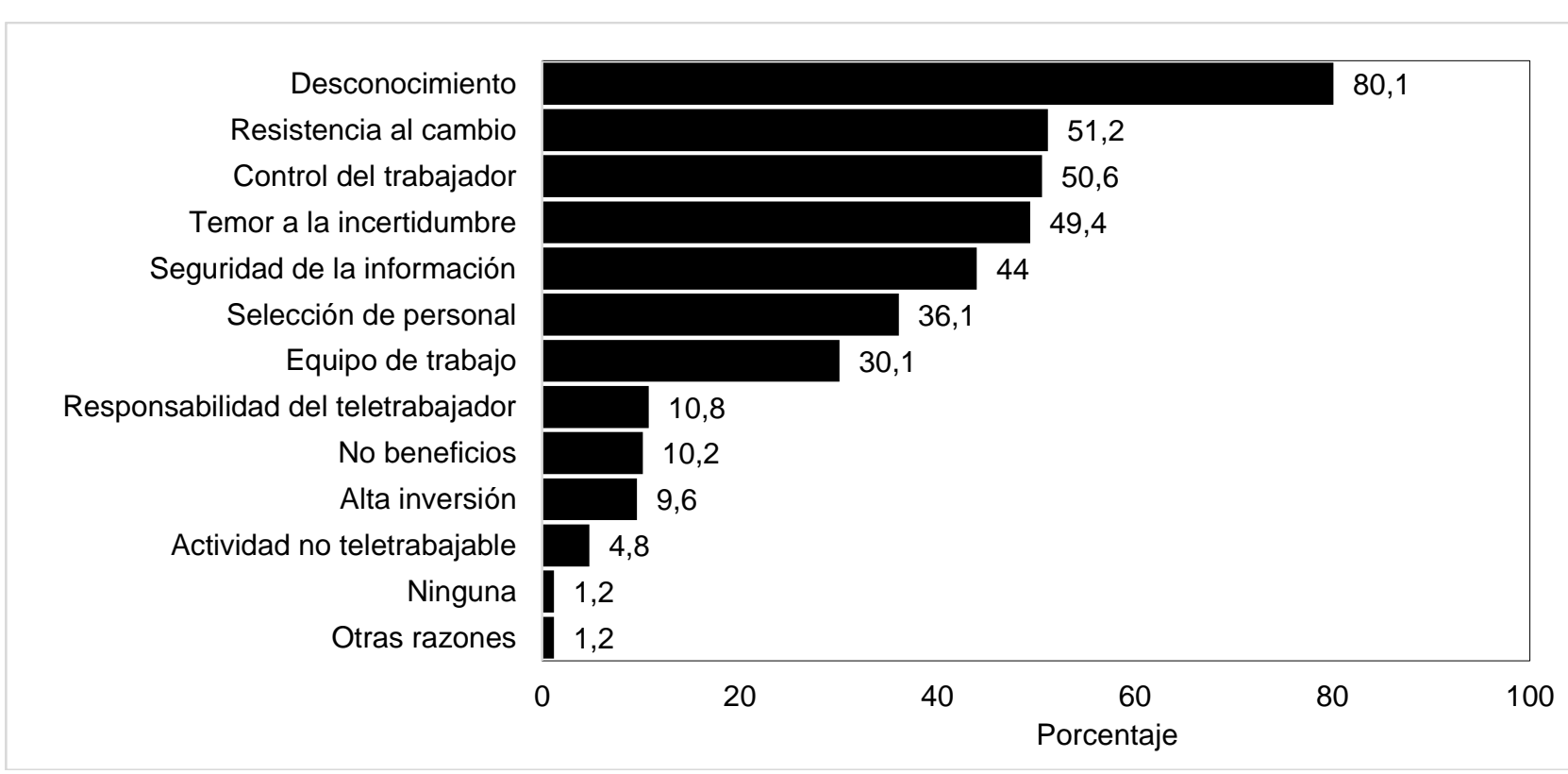

Fig. 2: Dificultades señaladas como las más importantes para adoptar el teletrabajo

Pasando al análisis multivariado, los resultados del ACM arrojan que las dos primeras dimensiones retienen $52.7 \%$ de la variabilidad contenida en los datos, que aunque relativamente bajo, es coherente con los resultados obtenidos en estudios con variables categóricas, además, como queda registrado en la Tabla 3 , la variable de interés, disposición hacia el teletrabajo, queda bien representada en este primer plano factorial, dado que carga en ambas dimensiones del mismo.

Tabla 3: Coordenadas de las variables categóricas en el primer plano perceptual del ACM

\begin{tabular}{|c|c|c|c|}
\hline \multirow{2}{*}{ Variables } & \multicolumn{2}{|c|}{ Dimensión } & \multirow{2}{*}{ Media } \\
\cline { 2 - 3 } & 1 & 2 & 0,148 \\
\hline Número Trabajadores & 0,10 & 0,18 & 0,08 \\
\hline Nube & 0,14 & 0,01 & 0,05 \\
\hline Capacitación & 0,10 & 0,00 & 0,21 \\
\hline Monitoreo & 0,01 & 0,41 & 0,13 \\
\hline Decisiones & 0,26 & 0,00 & 0,10 \\
\hline Supervisor & 0,01 & 0,18 & 0,22 \\
\hline Supervisión Directa & 0,44 & 0,01 & 0,14 \\
\hline Riesgos Seguridad & 0,08 & 0,21 & 0,18 \\
\hline Cambiar Forma Trabajar & 0,35 & 0,00 & 0,22 \\
\hline Trabajo Visible & 0,44 & 0,00 & 0,16 \\
\hline Negocios & 0,05 & 0,26 & 0,34 \\
\hline Disposición Teletrabajo & 0,27 & 0,40 & 1,96 \\
\hline Total activo & 2,25 & 1,67 & 16,33 \\
\hline \% de la varianza & 28,77 & 23,89 &
\end{tabular}

En la misma tabla se observa además como las variables que atañen al uso de herramientas de la nube, la realización de cursos de capacitación en informática para los empleados, el empoderamiento a los empleados para la toma de decisiones, el manifestarse sobre la supervisión directa necesaria para el seguimiento de las actividades laborales de los empleados y la postura que se tiene sobre cambiar la forma de trabajar, cargan predominantemente hacia la dimensión 1; mientras que el tamaño de la empresa, el disponer de software 
para el monitoreo de las actividades laborales, la existencia de la figura de supervisor, el manifestar que existe riesgo para mantener la seguridad de la información cuando el trabajador labora desde su domicilio, el declarar ser confiado o cuidadoso cuando realiza negocios con personas desconocidas, cargan en mayor intensidad a la dimensión 2.

En el mapa perceptual obtenido en el ACM (Figura 3) se observa como las distintas modalidades de respuesta de la variable que nos interesa describir "Disposición hacia el teletrabajo" quedan localizadas casi que en los cuatro puntos cardinales del mapa, discriminando de izquierda a derecha, los empresarios donde se ha implementado el teletrabajo (SiTT) y los que no manifiestan ninguna intención de hacerlo (NoTT), y de arriba hacia abajo, los que saben qué es el teletrabajo pero no planean implementarlo a corto plazo (NoTTcp) y los que no conocían el teletrabajo pero que después de enterarse del tema quizás considerarían implantarlo en el futuro (QuizasTT).

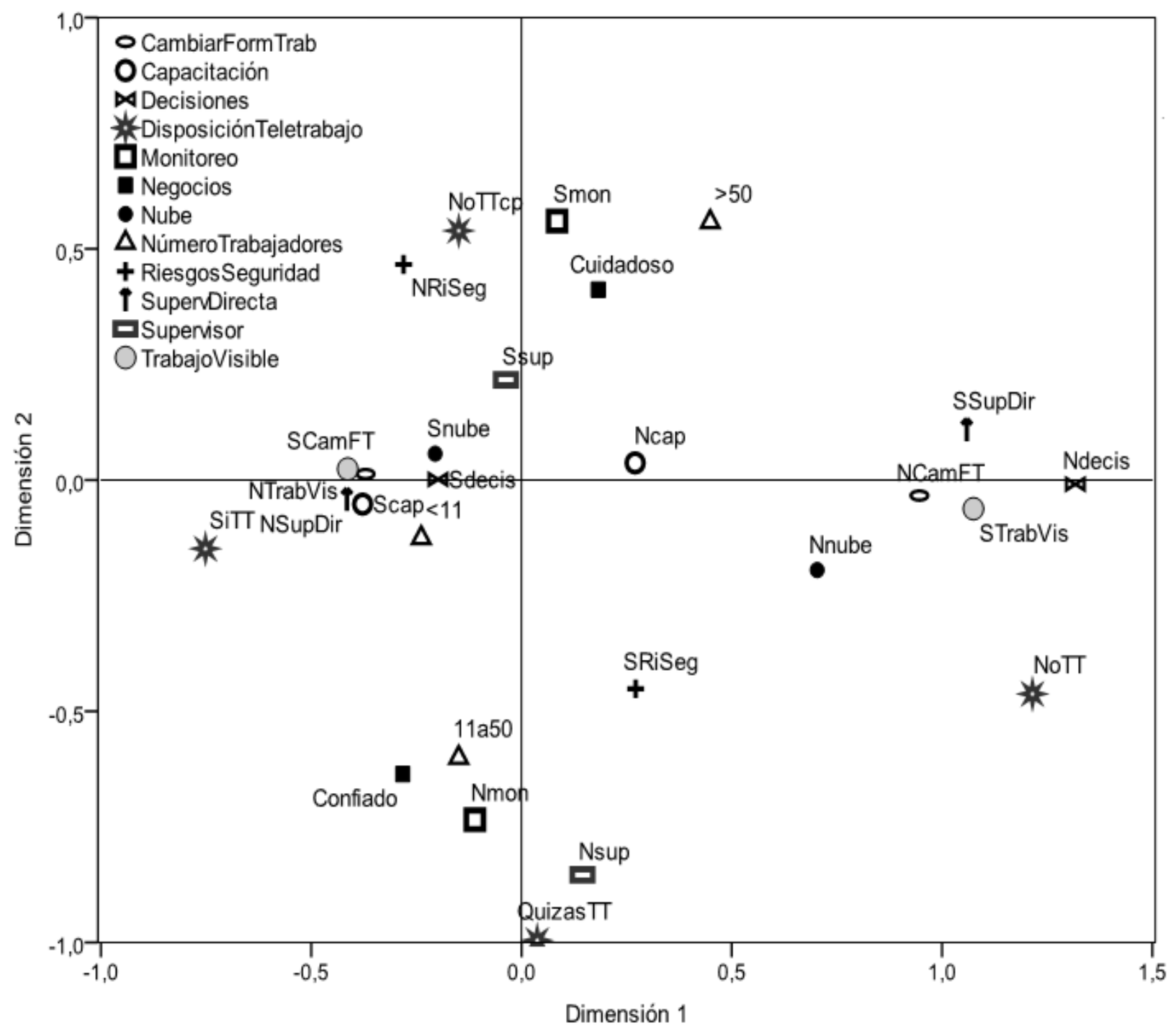

Fig. 3: Mapa perceptual obtenido del ACM

Dado lo anterior, se podría esbozar unos perfiles basados en las respuestas obtenidas a saber: los directivos de empresas en las que se ha implementado el teletrabajo están asociados a respuestas tales como si haber realizado cursos de capacitación en el último año en competencias informáticas a sus empleados (Scap), si tener disposición a cambiar su forma de trabajar (SCamFT), no estar de acuerdo con que la evaluación de los talentos sea un desafío sin supervisión directa (NSupDir), ni que el trabajo deba ser visible para su supervisión (NTrabVis), el proporcionar empoderamiento a sus empleados para la toma de decisiones (Sdecis), el pertenecer predominantemente a empresas pequeñas $(<11)$ y el hacer uso de herramientas de almacenamiento en la nube (Snube).

Al respecto Pérez et al. (2003) expresan que dado que las organizaciones son cada vez más conscientes de la importancia del conocimiento y el aprendizaje para obtener una ventaja competitiva, y consecuentemente incorporan con más frecuencia cambios técnicos y cambios organizativos, se muestran más acostumbradas 
a trabajar en entornos de alta incertidumbre, por lo que las empresas con programas de formación para empleados perciben una mayor viabilidad de acoger dicha forma de organización laboral. En este sentido, los resultados alcanzados por Arévalo et al. (2018) revelan que mejoras en las prácticas administrativas de las firmas acompañadas de inversiones en capacitación de personal, tecnologías de información y gestión del conocimiento, presentan una influencia significativa y positiva en la productividad y rentabilidad de las firmas. Asimismo, Peters y Dulk (2003) exponen que las organizaciones que tienen distanciamientos cortos de poder entre directivos y empleados, son más propensas a adoptar configuraciones de trabajo flexible, en las que la autonomía y empoderamiento del trabajador son rasgos característicos.

En contraposición, se localizan los empresarios que manifiestan no tener intención de aceptar el trabajo a distancia en sus empresas (NoTT), con todas las características antes mencionadas en modalidades de respuesta contrarias. Lo anterior está en concordancia con lo expresado por Peters (2015), quien afirma que la cultura organizacional puede ser un factor estimulante o restrictivo en las decisiones para acoger nuevos esquemas de trabajo de las organizaciones. Particularmente, enfoques administrativos en los que los componentes relacionales se centran en el control directo y el contacto cara a cara pueden considerarse como una barrera en este aspecto. En el mismo sentido, Dickson y Clear (2006) expresan que impulsados por el temor a que los empleados puedan hacer o dejar de hacer cuando no se encuentran a la vista, persiste entre muchos directivos una predilección hacia la supervisión física, lo que conduce a una postura de desconfianza hacia el empleado, y sin tal confianza existirán pocas probabilidades de que un proceso de puesta en marcha de estrategias de trabajo remoto resulte exitoso. Por su parte, Raghuram y Fang (2014) manifiestan que algunos directivos perciben un efecto negativo del distanciamiento espacial en el control y la supervisión que se puede ejercer sobre los subordinados, de forma tal que al incrementarse la distancia, los teletrabajadores son más renuentes a buscar instancias de información y retroalimentación de sus supervisores, así como los mismos supervisores encuentran una mayor dificultad para monitorear y evaluar el desempeños de sus empleados.

Entre tanto, en dirección vertical, se localizan en la parte superior del mapa, los empresarios que declaran saber qué es el teletrabajo pero que no piensan implementarlo a corto plazo (NoTTcp) altamente asociados a respuestas como el no considerar que el tener a sus empleados trabajando desde sus casas sea un riesgo para la seguridad de la información de la empresa (NRiSeg), el disponer de software para el monitoreo de las actividades laborales (Smon), el manifestar ser una persona cuidadosa cuando realiza negocios con desconocidos (Cuidadoso), el tener la figura de supervisor en la empresa (Ssup) y el laborar en empresas predominantemente grandes $(>50)$. El perfil anterior, podría explicarse desde el hecho de que la empresa posiblemente no esté motivada a poner en funcionamiento estrategias de trabajo a distancia en su organización dado que no están presentes ninguno de los desencadenantes que conlleven a contemplar dicha modalidad laboral, según lo describen lllegems y Verbeke (2004). Desencadenantes tales como desempeñarse en un sector basado en el conocimiento, que sus instalaciones se encuentren localizadas en un área de alta congestión vehicular, con un alto nivel de comunicación electrónica, enfocada en la toma de decisiones no rutinarias, con experiencia en el uso de horas de trabajo flexible, que realicen labores que impliquen poca interacción o baja demanda de trabajo colaborativo, entre otros.

En oposición se ubican los directivos que manifestaron no saber qué era el teletrabajo, pero que después de enterarse estarían dispuestos en adoptarlo en el futuro, asociado a respuestas como el no disponer de supervisor ni de software para el monitoreo de las actividades laborales, el declarar ser una persona confiada cuando realiza negocios con desconocidos, el laborar en empresas medianas ( 11 a 50) y el no considerar que hayan riesgos de seguridad en la información relacionados con trabajadores que realicen sus labores desde sus hogares. Lo anterior es coherente por lo enunciado por Kirs y Bagchi (2012), cuando exponen que aunado a otros factores relevantes, la confianza emerge como una condición necesaria previa a la adopción de las herramientas TIC, tanto a nivel empresarial como gubernamental. Lo anterior está alineado con lo expresado en múltiples investigaciones, en cuanto a que la aceptación del trabajo a distancia requiere del cambio en las posturas organizacionales conducentes a darle una mayor empoderamiento al empleado, mayor autonomía en sus decisiones y un cambio en las estrategias de evaluación del desempeño menos direccionada al seguimiento de actividades y cumplimiento de horarios a otro más centrado al cumplimiento de metas y objetivos (Dickson y Clear, 2006; Turetken et al., 2011;Topi, 2004; Davis, 2012).

\section{CONCLUSIONES}

De los resultados obtenidos, surge como principal dificultad para la aceptación del trabajo a distancia, en un contexto regional, una muy poco referenciada en la literatura, como es el desconocimiento entre directivos del concepto y de las políticas gubernamentales de fomento al teletrabajo. Entre las otras razones manifestadas se destacan algunas que aluden no a barreras tecnológicas y de inversión sino a aspectos de gestión administrativa, tales como las posturas directivas hacia aspectos de tipo organizacional, la resistencia al cambio, el temor hacia la incertidumbre, la pérdida de control de los empleados, el riesgo para la seguridad 
en la información, la posible incidencia en la dinámica al interior de los equipos de trabajo y los cambios a realizar en los procesos de selección de personal para la detección de nuevos perfiles más acordes a esta modalidad laboral. Igualmente, se detectan marcadas diferencias entre los directivos de empresas que han implementado el teletrabajo y las que no han considerado hacerlo, destacándose que los primeros, a diferencia de los segundos, dirigen organizaciones que con frecuencia realizan capacitaciones en competencias informáticas para sus empleados, presentan una alta disposición a cambiar su forma de trabajar, permiten la participación de sus empleados en la toma de decisiones laborales, hacen uso de herramientas de almacenamiento en la nube y no ven mayores dificultades en monitorear las actividades de sus empleados cuando realizan su labor en forma remota.

\section{AGRADECIMIENTOS}

Los autores agradecen a la Universidad Nacional de Colombia Sede Manizales a través de la dirección de investigaciones (DIMA) por el apoyo institucional y financiero al desarrollo de esta investigación (proyecto: "Modelo de medición de la productividad para teletrabajadores de empresas del sector servicios en Manizales" código Hermes 36757).

\section{REFERENCIAS}

Ahmed, A., A. Ishaque y otros tres autores, Telecommuting: Impact on Productivity of Telecommuters, doi: 10.1109/ICMIT.2014.6942423, Proceeding of the IEEE ICMIT (2014)

Arévalo-Avecillas, D., S. Nájera-Acuña y E. Piñero, La Influencia de la Implementación de las Tecnologías de Información en la Productividad de Empresas de Servicios, Inf. Tecnol., 29(6), 199-212, https://dx.doi.org/10.4067/S071807642018000600199 (2018)

Bailey, D. E. y N.B. Kurland, A Review of Telework Research: Finding, New Directions, and Lessons for the study of Modern Work, doi: 10.1002/job.144, Journal of Organizational Behavior, 23(1), 383-400 (2002)

Botzoris, G.N., V.A. Profillidis y A.T. Galanis, Teleworking and Sustainable Transportation in the Era of Economic Crisis, doi: 10.18638/ictic.2016.5.1.295, The 5th International Virtual Scientific Conference on Informatics and Management Sciences, March, 21-25, (2016)

Bloom, N., J. Liang, J. Roberts y Z. Ying, Does Working from Home Work? Evidence from a Chinese Experiment, Quarterly Journal of Economics 130(1), 165-218 (2014)

Burbach, M., E. y F.C. Day, Does Organization Sector Matter in Leading Telework Teams? A Comparative Case Study, doi: 10.1037/mgr0000014, International Journal of Business Information and Technology, 1(1), 29-42 (2012)

CCIT-Fedesarrollo, Teletrabajo: un Vistazo al Caso Colombiano, Coyuntura TIC, http://www.ccit.org.co/estudios/teletrabajo-un-vistazo-al-caso-colombiano/, 3-13 (2014).

Coenen, M. y R.A. Kok, Workplace Flexibility and New Product Development Performance: The Role of Telework and Flexible Work Schedules, doi: 10.1016/j.emj.2013.12.003, European Management Journal, 32(1), 564-576 (2014)

Corporación Colombia Digital - Ministerio TIC, Cuarto estudio de penetración de teletrabajo en empresas colombianas 2018, www.teletrabajo.gov.co/622/w3-article-75985.html, (2018)

DANE-Departamento Administrativo Nacional de Estadística, Clasificación Industrial Internacional Uniforme de todas las actividades económicas, Revisión 3.1 adaptada para Colombia-CIIU Rev. $3.1 \quad$ A. C., https://www.dane.gov.co/files/correlativas/CIIU_rev_3_1_parl.pdf, (2006)

Davis, A., Telework Productivity and Effectiveness: Factors that Influence Results Oriented Job Assessments, University of Oregon, https://scholarsbank.uoregon.edu/xmlui/bitstream/handle/1794/11385/Davis-2011. pdf?sequence=1, (2012)

Dickson, K. y F. Clear, Management Issues in the Adoption of Telework amongst SMEs in Europe, doi: 10.1109/PICMET.2006.296745, PICMET Proceeding, 9-13 July, Istanbul, Turkey: 1703-1708 (2006)

Golden, T. D., Altering the Effects of Work and Family Conflict on Exhaustion: Telework during Traditional and Nontraditional Work Hours, doi: 10.1007/s10869-011-9247-0, Journal of Business \& Psychology, 27(3), 255-269 (2012)

Golden, T.D. y J.F. Veiga, The impact of superior-subordinate relationships on the commitment, job satisfaction, and performance of virtual workers, doi: 10.1016/j.leaqua.2007.12.009, The Leadership Quarterly, 19(1), 77-88 (2008)

Harker, B.M. y R. MacDonnell, Is Telework Effective for Organizations? A meta-analysis of empirical research on perceptions of telework and organizational outcomes, doi: 10.1108/01409171211238820, Management Research Review, 35(7), 602-616 (2012).

Illegems, V. y A. Verbeke, Telework: What does it Mean for Management? doi: 10.1016/..lrp.2004.03.004, Long Range Planning, 37, 319-334 (2004)

Kim, H.W., Design of Information System Audit Model for Information Security in Smart Work Environment, ISSN: 1816949X, J. Eng. Applied Sci, 13 (Special Issue 2): 3042-3047 (2018) 
Kirs, P. y K. Bagchi, The Impact of Trust and Changes in Trust: A National Comparison of Individual Adoptions of Information and Communication Technologies and Related Phenomenon, doi: 10.1016/j.ijinfomgt.2012.02.003, International Journal of Information Management, 32, 431-441 (2012)

Kossek, E. E., B.A. Lautsch, S.C. Eaton, Telecommuting, Control, and Boundary Management: Correlates of Policy Use and Practice, Job Control, and Work-Family Effectiveness, doi: 10.1016/j.jvb.2005.07.002, Journal of Vocational Behavior. 68(2), 347-367 (2006)

Mello, J. A., Managing Telework Programs Effectively, doi: 10.1007/s10672-007-9051-1, Employ Respons Rights J. (19), 247-261 (2007)

Neufeld, D., Z. Wan y Y. Fang, Remote Leadership, Communication Effectiveness and Leader Performance, doi: 10.1007/s10726-008-9142-x, Springer Science, 19(1), 227-246 (2010)

Pearce II, J. A., Successful Corporate Telecommuting with Technology Considerations for Late Adopters, doi: 10.1016/j.orgdyn.2008.10.002, Organizational Dynamics, 38(1), 16-25 (2009)

Pérez, S. C., El teletrabajo: ¿Más libertad o una nueva forma de esclavitud para los trabajadores? http://www.redalyc.org/articulo.oa?id=78817024008, Revista de Internet, Derecho y Política, (11), 24-33 (2010)

Pérez, M., A. Sánchez y P. Carnicer, The Management of Teleworking Adoption: An Organisational Study of Feasibility Perception in Industrial and Service Companies, doi:10.1504/ijhrdm.2003.003663, International Journal of Human Recources Development and Management, 3(3), 226-248 (2003)

Pérez, M., A. Martínez, P.L. Carnicer y M.J. Vela, The Differences of Firm Resources and the Adoption of Teleworking, doi: 10.1016/j.technovation.2005.04.002, Technovation, 25(1), 1476-1483 (2005)

Peters, P., R. Batenburg, Telework adoption and Formalisation in organizations from a knowledge transfer perspective, http://hdl.handle.net/2066/155442, Int. J. Work Innovation, 1(3), 251-270 (2015)

Peters, P. y L. den Dulk, Cross Cultural Differences in Managers' Support for Home-based Telework, doi: 10.1177/1470595803003003005, International Journal of Cross Cultural Management, 3(3), 329-346 (2003)

Peters, P., K.G. Tijdens y C. Wetzels. Employees' Opportunities, Preferences, and Practices in Telecommuting Adoption, doi: 10.1016/S0378-7206(03)00085-5, Information \& Management, 4(1), 469-482 (2004)

Raghuram, S. y D. Fang, Telecommuting and the Role of Supervisory Power in China, doi: 10.1007/s10490-017-9546-8, Asia Pac J Manag., (3), 523-547 (2014)

Topi, H., Supporting Telework: Obstacles and Solutions, doi: 10.1201/1078/44432.21.3.20040601/82481.12, Information Systems Management, 21(3), 79-85 (2004)

Turetken, O., A. Jain, B. Quesenberry y O. Ngwenyama, An Empirical Investigation of the Impact of Individual and Work Characteristics on Telecommuting Success, doi: 10.1109/TPC.2010.2041387, IEEE Transactions on Professional Communication, 54(1), 56-67 (2011)

Valmohammadi, C, Investigating the Perceptions of Iranian Employees on Teleworking. Industrial and Commercial Training, doi: 10.1108/00197851211231513, Emerald Insight, 44(4), 236-241 (2012) 